\title{
LES DIFFÉRENTS ENJEUX DE LA CITOYENNETÉ SEXUELLE SELON LA CONFIGURATION GÉOPOLITIQUE: UNE ANALYSE COMPARATIVE ENTRE LE BRÉSIL ET LES ÉTATS-UNIS
}

\section{BARBARA ANDRADE DE SOUSA}

Université du Québec à Montréal

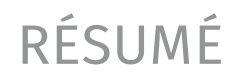

L'article se divise en deux analyses complémentaires. La première examine les différences entre la tentative de la construction de la citoyenneté sexuelle aux États-Unis et au Brésil. L'exposition comparative des demandes des mouvements sociaux des gays et des lesbiennes des deux pays permet la mise en évidence que chaque contexte social et politique génère des enjeux distincts et que, par conséquent, la quête de la construction de la citoyenneté sexuelle ne peut se faire de la même manière. Par la suite, l'article se concentre sur le Brésil et s'interroge sur la possibilité d'avoir une citoyenneté sexuelle réussie dans un pays qui présente des taux alarmants de violence contre la population LGBT. Étant donné la pénurie d'analyses sur ce sujet, l'article cherche à élucider telle relation en mobilisant les études qui traitent du lien entre la violence et la démocratie, ainsi que ceux qui placent le féminicide et le racisme comme barrières d'accès à la pleine citoyenneté.

Mots-clés: Citoyenneté sexuelle. LGBTQ. Violence.

\section{ABSTRACT THE DIFFERENT ISSUES OF SEXUAL CITIZENSHIP} \section{ACCORDING TO GEOPOLITICAL ARRAY: A COMPARATIVE}

\section{ANALYSIS BETWEEN BRAZIL AND THE UNITED STATES}

This article is divided into two complementary analysis. The first one aims to appraise the dissimilarities in the attempt of constructing a sexual citizenship in Brazil's and the US's context. The comparative exposition of civil movements championed by gays and lesbians in both societies brings to light that each particular geographic and political context stems its own demands and thus the formation of the sexual citizenship occurs disparately. Following that, the article focus on Brazil and inquires whether it is feasible for a sexual citizenship to be actually formed in a country that displays alarming rates 
of violence towards the LGBTQ community. As studies on such topic are rare, the present article aspires to shed light into the matter by means of analysis of articles that dwell on the connection between violence and democracy as well as those that place feminicide and racism as barriers for a full access to citizenship.

Keywords: Sexual citizenship. LGBTQ. Violence.

\section{RESUMO AS DIFERENTES QUESTÕES DA CIDADANIA SEXUAL DE ACORDO COM A CONFIGURAÇÃO GEOPOLÍTICA: UMA ANÁLISE COMPARATIVA ENTRE BRASIL E ESTADOS- UNIDOS}

O artigo divide-se em duas análises complementares. A primeira busca examinar as diferenças, na tentativa de construção da cidadania sexual, no contexto do Brasil e dos Estados Unidos. A exposição comparativa das lutas dos movimentos sociais de gays e lésbicas dessas duas sociedades permite evidenciar que cada contexto geográfico e político específico gera reivindicações distintas e, consequentemente, a busca da construção da cidadania sexual não acontece da mesma maneira. Em seguida, o artigo concentra-se no Brasil e questiona a possibilidade da existência de uma cidadania sexual nesse país que apresenta índices alarmantes de violência contra as pessoas LGBTQ. Sendo raros os estudos sobre a temática, o artigo procura elucidar tal relação utilizando as análises que tratam do nexo entre violência e democracia, assim como as que colocam o feminicídio e o racismo como barreiras para o acesso à cidadania plena.

Palavras-chave: Cidadania sexual. LGBT. Violência.

\section{Introduction}

La citoyenneté a longtemps été considérée comme un instrument de la loi. Depuis le travail de Marshall (1950), qui a établi un pont entre la citoyenneté et la sociologie, les études sur la citoyenneté se sont multipliées. Ce champ d'études comporte de multiples ramifications, la citoyenneté sexuelle en étant une. Le « sexuel " a toujours été considéré comme appartenant au domaine de la vie privée, tandis que la citoyenneté faisait partie de la sphère publique. Le développement de ce domaine d'études a défié la séparation clas- sique existante entre ces deux sphères, qui a toujours constitué la base de la citoyenneté (LISTER, 2002). Le terme « citoyenneté sexuelle » est un parapluie qui abrite différents aspects à l'intérieur du thème plus large du corps et de la sexualité. Autrement dit, il y a des études qui s'inscrivent dans le champ de la citoyenneté sexuelle et qui analysent le droit à l'avortement, le rapport entre les genres, l'accès que les personnes homosexuelles ont aux droits civils de base, etc. (LISTER, 2002). Cet article se concentre sur les aspects de la citoyenneté 
sexuelle relatifs aux personnes de la diversité sexuelle.

Principalement analysé dans les contextes du Nord global, ce concept semble ne pas rendre compte des spécificités auxquelles sont confrontées les personnes de la diversité sexuelle qui habitent dans les pays du Sud global. En effet, nous remarquons qu'il y a de plus en plus de recherches récentes qui ont constaté cette pénurie d'analyses et s'interrogent sur la construction de la citoyenneté sexuelle dans d'autres contextes (RICHARDSON, 2017; PLUM$M E r$, 2005). Le présent travail cherche donc à combler cette lacune par le biais d'une analyse comparative entre le contexte états-unien et celui brésilien à travers laquelle nous démontrons la différence en ce qui concerne la construction de la citoyenneté sexuelle dans ces deux pays.

La pertinence de la prise en compte d'autres contextes pour l'analyse de la citoyenneté sexuelle réside dans la découverte de nouveaux aspects qui y sont liés. Dans le cas du Brésil, la violence commise contre les personnes LGBT peut être considérée comme étant une barrière qui empêche le vécu de la citoyenneté par cette partie de la population. L'apport de cet article réside dans l'investigation de la relation entre citoyenneté sexuelle et violence, une relation qui a été mise en exergue avec la violence faite aux femmes et aux personnes noires, mais rien n'a été trouvé sur la citoyenneté et la violence exercée à l'endroit des personnes de la diversité sexuelle dans les pays qui possèdent un haut niveau de crime de haine contre cette partie de la population.

\section{De la citoyenneté à la citoyenneté sexuelle}

Le point de départ de la relation entre la sociologie et la citoyenneté est souvent retra- cé dans l'œuvre de T.H. Marshall, sociologue britannique qui, en 1950, a écrit « Citizenship and Social Class ». Dans ce livre, qui s'avère centré sur la situation de son pays d'origine, Marshall identifie trois dimensions de la citoyenneté, chacune composée des institutions de la société moderne : la dimension civile, qui est constituée des systèmes légaux; la dimension politique représentée par le gouvernement démocratique et la dimension sociale composée du système de protection sociale (Roche, 2002). Dans le cadre de cet article, la dimension qui nous intéresse est surtout celle sociale. Il est donc important de préciser que pour Marshall, cette dimension s'étend du droit à un minimum de bien-être économique et social au droit de vivre de manière civilisée, conformément aux normes en vigueur dans la société.

Depuis l'étude de Marshall, la sociologie de la citoyenneté a connu de nombreuses œuvres remarquables. Le concept de citoyenneté fut décortiqué en plusieurs aspects qui n'ont pas été analysés par Marshall, mais qui s'inspirent du caractère dynamique qu'il a attribué à la citoyenneté. Par exemple, le livre de Isin et Turner (2002) sur les études sur la citoyenneté compte huit chapitres qui décrivent l'analyse de différentes formes de citoyenneté comme la culturelle, l'environnementale, la religieuse et la sexuelle, pour ne pas toutes les nommer. Ces études démontrent l'existence de certains individus qui ont le statut légal de citoyens, mais qui ne disposent pas d'une citoyenneté sociale complète. L'intérêt de la sociologie en ce qui a trait à l'étude de la citoyenneté réside dans le processus social à travers lequel des barrières entravent, pour certains individus, le plein vécu de leur citoyenneté (GLENN, 2011).

Selon Hall et Held (1989), l'analyse des formes contemporaines de la citoyenneté doit prendre en compte le rôle des mouvements sociaux dans l'expansion des revendications des 
droits et privilèges à de nouveaux domaines sociaux, comme les droits des femmes, la lutte contre le racisme et la xénophobie, etc. Les auteurs défendent l'idée que, pour obtenir une compréhension actuelle de la citoyenneté, on doive se pencher sur l'imbrication des multiples identités et des identifications existantes au sein de la société moderne, sur le rôle de la citoyenneté vis-à-vis ces enjeux identitaires complexes ainsi que sur les différentes formes de participation à la vie sociale.

Le Gay Liberation, mouvement social des années soixante-dix, dont les protagonistes étaient les hommes gays américains, visait à renverser l'ordre existant (D'EMILIO, 1998; SOUSA, 2016). L'orientation sexuelle devrait être motif de fierté, et non pas de honte, donc leur slogan disait « gay is ok ». Auparavant, être gay était synonyme d'anonymat, de vie privée. L'objectif du Gay Liberation était d'amener la discussion dans le domaine public et, pour ce faire, ils ont réclamé la sortie du placard des homosexuels et lesbiennes américains, dans le but de montrer leur nombre et leur insatisfaction en lien avec la répression policière. Ce mouvement social a consolidé sa capacité de mobilisation collective au fil des années et a constitué un puissant groupe de lutte pour les droits des gays et lesbiennes américains (DUGGAN, 2004). C'est dans cet axe identitaire que se situe le champ de la citoyenneté sexuelle.

La théorie sur la citoyenneté sexuelle a pris son essor dans les années quatre-vingt-dix (EVANS, 1993; PLUMMER, 1995; RICHARDSON, 1998; WEEKS, 1998). La théoricienne britannique Diane Richardson est une des spécialistes de l'étude de la citoyenneté sexuelle, ayant écrit plusieurs articles sur le sujet. Dans l'un de ses articles, Richardson (2000) divise les analyses de la citoyenneté sexuelle en deux domaines différents. D'abord, on trouve les études sur les droits liés à la sexualité et la lutte pour leur acquisition qui désignent les droits sexuels accordés ou refusés à divers groupes sociaux. Pour les gays et lesbiennes du Nord global, les luttes plus emblématiques concernaient le droit à l'union civile et le droit de servir dans l'armée, un droit qui, dans la plupart des législations au monde, est intrinsèquement lié à la citoyenneté : seuls les citoyens peuvent y servir. Ensuite, il y a les analyses qui se penchent plus amplement sur la société et les implications théoriques de l'accès ou de l'exclusion de certains droits fondés sur la sexualité et qui démontrent comment les différentes formes du statut de citoyen dépendent de la sexualité de l'individu.

Comme mentionné ci-dessus, l'essor de ces études a eu lieu dans les années quatre-vingtdix, époque où la majorité des pays du Nord global n'avaient pas encore accordé le droit à l'union civile aux personnes du même sexe. Or, l'acquisition de ce droit et aussi du droit de servir dans l'armée n'a pas mis fin aux études sur la citoyenneté sexuelle, comme le démontre un autre article de Richardson (2017) où, encore une fois, l'auteure révise la littérature sur ce sujet. Cette fois-ci, elle disserte sur d'autres domaines qui se sont développés à l'intérieur de la théorie sur la citoyenneté sexuelle au long des années. Dans la conclusion de cet article, Richardson soulève la question de la construction de la citoyenneté sexuelle à travers différents contextes géopolitiques. Nous voyons alors que ce champ d'études demeure effervescent, et ce travail cherche à élargir la discussion au Sud global, plus précisément au contexte brésilien.

Si, comme le défendent Hall et Held (1989), l'analyse des formes contemporaines de la citoyenneté doit être réalisée dans une perspective qui examine le rôle des mouvements sociaux dans l'expansion des revendications des droits et privilèges à de nouveaux domaines, nous postulons que le contexte géopolitique est sans doute déterminant en ce qui concerne 
les revendications portées par un groupe donné. L'une des principales demandes des mouvements des gays et des lesbiennes américains et canadiens a été la décriminalisation de l'homosexualité (CARTER, 2005; D'EMILIO, 1998; DEMCZUK, 1998; HIGGINS, 2011). Or, au Brésil, depuis 1830, les références à la sodomie ne font plus partie du Code pénal (FACCHINI et ASSIS, 2009). D'autres facteurs ont alors guidé les revendications de ce groupe, comme l'on verra plus tard.

Il y a de plus en plus de chercheurs et de chercheures qui problématisent l'approche colonisatrice et impérialiste du transfert de savoir du Nord au Sud (REA et AMANCIO, 2018; PELUCIO, 2012; PEREIRA, 2015; SPIVAK, 2009). D'autres s'interrogent à savoir si certaines théories produites et pensées au Nord peuvent être applicables au Sud, sans qu'il n'y ait une imposition de connaissance qui annihile les contextes locaux (RICHARDSON, 2017; PLUMMER, 2005; MIÑOSO, 2015; EL TAYEB, 2011). Sans nous attarder à la littérature sur ce sujet qui est vaste, nous voulons simplement attirer l'attention sur l'importance d'une production académique qui valorise la réflexion sur la prise en compte des facteurs et des connaissances locales dans l'analyse d'un phénomène donné, sans pour autant imposer un cadre théorique qui ne peut pas rendre compte des spécificités locales.

Ainsi, ce travail propose une analyse comparative entre le contexte du Brésil et du Nord global, en ce qui concerne une pleine participation à la citoyenneté des personnes de la diversité sexuelle. Autrement dit, quelles sont, selon les contextes géopolitiques, les barrières qui limitent ou qui empêchent l'accès à certains droits en raison de l'orientation sexuelle des citoyens? Pour réaliser cette comparaison, nous nous inspirons de certaines questions soulevées par les auteurs qui ont travaillé sur ce sujet (RICHARDSON, 2017; PLUMMER, 2005), soit la construction de la citoyenneté sexuelle dans le Sud global. Le but n'est pas de savoir si le concept de la citoyenneté sexuelle telle que pensée dans le Nord est applicable au Sud, mais plutôt de nous baser sur ce concept pour analyser la construction de cette catégorie au Brésil, sans pour autant imposer ce qui a déjà été analysé dans un contexte qui n'est pas le même.

\section{Méthodologie}

La démonstration de la construction de la citoyenneté sexuelle au Brésil ne peut pas être faite sans une analyse sociohistorique des facteurs qui ont stimulé et modelé les demandes du mouvement des gays et lesbiennes dans ce pays. Après avoir dressé le portrait de ce mouvement social, nous montrerons si leurs demandes ont été adressées, si la construction de la citoyenneté sexuelle s'est faite exclusivement à partir de ces demandes ou s'il y a d'autres facteurs qui empêchent ou limitent l'adhésion à la société dans laquelle ils vivent et aux droits et privilèges qui sont accordés aux Brésiliens hétérosexuels.

Comme cet article a aussi comme objectif de valider la construction d'une catégorie analytique qui a été conçue au Nord global dans le Sud global, une comparaison sera établie entre la situation américaine et celle du Brésil. Le choix des États-Unis comme unité d'analyse se justifie par le fait que la littérature sur la citoyenneté sexuelle dans le contexte américain est très riche et que ceci est un travail comparatif qui est basé sur la documentation existante. Le manque de littérature sur la question dans d'autres pays constitue l'une des limites de ce type d'analyse.

Pour répondre aux questions posées par cet article, quelques étapes ont été suivies, telles que préconisées par des auteurs qui se sont penchés sur la méthode d'analyse com- 
parative en sciences sociales. Nous combinons des éléments de la méthode d'histoire comparative avec ceux de l'étude de cas, qui comportent certaines ressemblances (RAGIN, 2014; RITTER, 2014), pour clarifier le phénomène analysé. L'étude comparative de cas fournit « une base pour examiner la manière dont les conditions se combinent de différentes manières et dans différents contextes pour produire des résultats différents » (Ragins, 2014 : p.45), tandis que l'analyse d'histoire comparative propose une méthodologie qui priorise une explication historiquement contextualisée et qui retrace l'émergence du phénomène considéré (RITTER, 2014).

Bien que l'analyse comparative entre les deux pays ne soit pas notre point central, d'autres travaux ont déjà démontré (CaNABARRO, 2013; IRINEU, 2014; ROSENEIL et STOILOVA, 2011) l'importance de cette contextualisation dans la compréhension du cheminement entrepris par le mouvement des gays et lesbiennes pour aboutir à la construction d'une citoyenneté sexuelle. Pour cette raison, la période analysée ne sera pas exhaustive. Nous allons réparer les principales revendications et accomplissements de ces mouvements sociaux depuis les années soixante-dix, époque où s'amorcent les premières tentatives de vivre l'homosexualité dans la sphère publique aux États-Unis (SOUSA, 2016). Au Brésil, c'est le moment où surgit le premier groupe de défense des droits des homosexuels. La comparaison sera effectuée pour démontrer la différence politique et sociale de ces contextes. Son objectif est de montrer que la construction de la citoyenneté sexuelle ne s'est pas faite de la même manière. Ensuite, on se penchera sur le contexte au Brésil qui n'a pas souvent été analysé.

Nous avons exploré la littérature existante sur la citoyenneté sexuelle dans les deux pays analysés et avons constaté une pénurie de littérature qui aborde ce thème au Brésil.
Malgré la rareté des études spécifiques sur ce concept, cela ne représente pas une limite parce qu'il y a une abondance de textes sur le mouvement des gays et lesbiennes (CANABARRO, 2013; IRINEU, 2014; FACCHINI et SIMÕES, 2009; TREVISAN, 2000), ce qui nous permet de démontrer les revendications du mouvement et la construction de la catégorie visée par cet article.

\section{Une brève histoire du mouvement homosexuel au Brésil}

C'est à la fin des années soixante-dix que l'on voit le premier groupe d'hommes gays se réunir pour penser l'homosexualité de façon politique. Le pays vivait encore sous la dictature militaire et l'idéologie du groupe était marquée par le contexte politique de l'époque. Ils misaient sur la contreculture, sur la contestation et ils étaient contre l'autoritarisme (CANABARRO, 2013; FACCHINI et SIMÕES, 2009). À l'instar de leur homologue états-unien, le regroupement brésilien Somos visait une transformation profonde dans la société et cherchait à se réapproprier et à mettre en valeur les termes utilisés de façon péjorative pour se référer aux gays et lesbiennes (FACCHINI et SIMÕES, 2009). L'éclosion de l'épidémie du SIDA dans les années quatre-vingt et l'affaiblissement du régime dictatorial changent inévitablement le caractère des revendications et le format de groupement du mouvement. Ainsi, dans cette décennie l'objectif de la militance devient le combat lié au SIDA et l'établissement d'un dialogue avec l'État qui se prépare à la réinstallation du régime démocratique.

À partir de cette époque, la militance du mouvement homosexuel ${ }^{1}$ au Brésil s'organise

1 À cette époque, le mouvement s'appelait « homosexuel ", mais il incluait les femmes lesbiennes et les travestis. L'appellation mouvement LGBT est beaucoup plus récente (GROSSI, 2017). Au Brésil, le mouvement utilise l'acronyme LGBT et non LGBTQ comme au Nord global. Nous nous alignons sur l'acronyme utilisé dans le pays. 
de façon institutionnelle et leurs revendications portent sur l'amélioration du système de santé pour les séropositifs, sur les demandes de droits civils et sur la lutte contre la discrimination et la violence que subissent les homosexuels (IRINEU, 2014; FACCHINI et SIMÕES, 2009). L'une des premières conquêtes du mouvement fut le retrait de l'homosexualité de la classification de maladies par le Conseil fédéral de médecine en 1985, cinq ans avant le changement réalisé par l'Organisation mondiale de la Santé. Cela s'est concrétisé grâce à une campagne d'envergure nationale qui a réuni plus de 16000 signataires (MOTT, 2005). Un an plus tard, on voit une tentative de dialogue entre le mouvement homosexuel et le pouvoir législatif pendant la période d'élaboration de la nouvelle constitution brésilienne. Certains groupes se sont mobilisés pour ajouter au texte constitutionnel l'interdiction de la discrimination fondée sur l'orientation sexuelle, ce qui a été barré par certains politiciens d'inclination religieuse (CANABARRO, 2013; IRINEU, 2014; FACCHINI et SIMÕES, 2009, MOTT, 2005).

Depuis les années quatre-vingt-dix, plusieurs projets de loi en faveur des droits des homosexuels ont été soumis au pouvoir législatif, mais ceux approuvés demeurent rares. Parmi les projets rejetés, on retrouve celui intitulé « Partenariat civil enregistré » entre personnes du même sexe qui remonte à 1995 et qui n'a jamais été voté, ainsi que le projet 122 de 2006 qui vise à criminaliser la discrimination basée sur l'orientation sexuelle. Ce dernier a été reformulé maintes fois et finit par être rejeté en 2013, ce qui représente une autre réussite des parlementaires d'inclination religieuse. La plupart des conquêtes du mouvement homosexuel viennent du pouvoir judiciaire, la plus importante étant la reconnaissance de l'union civile entre personnes du même sexe par le Tribunal suprême fédéral² en 2011.

2 La plus haute instance du pouvoir judiciaire brésilien.
Ce bref historique du mouvement homosexuel au Brésil expose leur inquiétude concernant la discrimination sociale et la violence que vivent les personnes de la diversité sexuelle au pays. Comme le défendent les sociologues Facchini et Simões (2009), l'activisme homosexuel brésilien insiste sur la dénonciation des violences spécifiques contre l'homosexualité. Les auteurs voient l'homophobie ${ }^{3}$ " comme un point d'ancrage à partir duquel se structurent les entités collectives associées au mouvement et se légitiment les revendications dans le domaine des droits et de la politique » (FACCHINI et SIMÕES 2009, p. 27). Aux ÉtatsUnis, les revendications du mouvement gay ne sont pas centrées sur des enjeux liés à la violence homophobe, comme on le verra sans tarder.

\section{Une brève histoire du mouvement gay aux États-Unis}

Une différence cruciale entre le contexte étatsunien et le brésilien, c'est qu'aux États-Unis, l'homosexualité était criminalisée. Initialement, le mouvement gay se structure pour combattre la violence policière, et non celle de la société tout entière, comme cela est le cas au Brésil. Les émeutes à Stonewall sont considérées comme étant les déclencheurs du mouvement gay dans ce pays. Ces émeutes sont une réponse à la répression policière. En effet, depuis des années, le corps policier harcelait la clientèle du bar Stonewall, situé à New York. En juin 1969, au lieu d'accepter l'intervention policière et de quitter le bar, les habitués ont décidé de manifester contre l'arrestation, ce qui a déclenché une vague de manifestations

3 Actuellement, des termes spécifiques sont utilisés pour parler de la violence exercée à l'endroit des personnes de la diversité sexuelle et de genre, comme : homophobie, lesbophobie, biphobie et transphobie. Dans le cadre de ce travail, afin d'alléger le texte, nous utilisons le terme " homophobie » comme parapluie aux violences subies par toutes les personnes appartenant à cette diversité. 
devant le bar dans les jours qui suivirent (CAR$T E R, 2005)$. C'était la première fois que les homosexuels contre-attaquaient et défiaient l'autorité institutionnelle ainsi que la répression de leur mode de vie.

Le mouvement connu sous le nom de Gay Liberation a pris son élan dans le climat soulevé par les émeutes de Stonewall. Ce mouvement, tout comme les autres ${ }^{4}$ qui ont surgi aux États-Unis dans les années soixante-dix, cherchait à dénoncer les structures sociales existantes pour les déconstruire et renverser les valeurs dominantes. Au lieu d'être gay et d'avoir honte, l'orientation sexuelle devrait être motif de fierté. Pour cette raison, ils ont encouragé la sortie du placard, ce qui représentait un changement crucial dans la façon d'être gay (D'Emilio, 1998). Auparavant, être gay était synonyme d'anonymat et de vie privée. Le but du Gay Liberation était d'amener la discussion au domaine public et de franchir la ligne entre le privé et le public. Ils ont ainsi déclenché un mouvement politique d'affirmation de soi dans lequel plus les gens sortaient du placard, plus d'autres gens désiraient en sortir.

Encore une fois, l'épidémie du SIDA a une influence dans les demandes et l'organisation du mouvement. Dans les années quatre-vingt, la communauté gay s'organise pour demander au pouvoir public une mobilisation rapide et efficace dans le combat contre l'épidémie (ARMSTRONG, 2002). Au fil des années, après l'éclosion et la stabilisation de la situation liée au SIDA, le mouvement des gays et lesbiennes concentre son attention sur les demandes relatives au mariage entre personnes du même sexe et l'interdiction aux personnes homosexuelles de déclarer leur orientation sexuelle lorsqu'elles servent dans l'armée (DUGGAN, 2004).

4 Notamment le woman liberation qui dénonçait le patriarcat et voulait s'en libérer et le Black Power qui cherchait à inverser le stigmate associé au fait d'être noir (D'EMILIO, 1983).
Institué en 1993, la politique sur la participation au service militaire des personnes de la diversité sexuelle, connue comme Don't ask, don't tell, prohibait le harcèlement dans l'armée de personnes homosexuelles ou bisexuelles qui ne divulguaient pas leur orientation sexuelle, tandis qu'elle interdisait à ceux et celles qui parlaient ouvertement de leur sexualité de rejoindre l'armée. Cette loi fut abrogée en 2011 et en 2015', après la décision de la Cour suprême, tous les États des ÉtatsUnis ont passé à reconnaitre le mariage entre personnes du même sexe.

\section{La relation entre violence interpersonnelle et citoyenneté sexuelle}

Nous avons vu les principaux enjeux qui ont marqué la lutte pour une pleine citoyenneté des personnes de la diversité sexuelle dans les deux pays analysés. Si, au Brésil, l'une des préoccupations principales demeure l'homophobie que subit cette partie de la population, aux États-Unis, ce problème semble ne pas faire partie des demandes du mouvement. Les chiffres concernant les personnes décédées à cause de l'homophobie au Brésil sont éloquents. Selon le rapport publié annuellement par le Groupe Gay da Bahia6 (GGB), en 2017, 445 personnes sont décédées en raison de leur orientation sexuelle, de leur identité ou de leur expression de genre. Ce chiffre représente une augmentation de 30 \% par rapport à 2016.

En vue des demandes de politiques publiques pour combattre l'homophobie au Brésil de la part des mouvements de gays et lesbiennes et des études qui démontrent l'homo-

5 Avant 2015, il y avait des États qui reconnaissaient le mariage gay. Pour plus d'informations, voir Paternotte et Tremblay (2015).

6 Association de défense des droits humains des homosexuels au Brésil qui publie depuis 1979 un rapport annuel des morts violentes des personnes LGBT dans le pays. Site web : https://grupogaydabahia.com.br/ 
phobie comme étant un composant indispensable dans la constitution du mouvement LGBT brésilien (FILHO, 2016; FACCHINI et SIMÕES 2009), nous proposons une analyse qui traite l'homophobie comme étant une barrière au plein vécu de la citoyenneté. D'autres études ont déjà montré que le féminicide (FRANZWAY, 2016; SORIANO, 2014) et le racisme (MITCHELL et WOOD, 1998; SMITH, 2015) constituent une barrière à l'exercice de la citoyenneté, mais aucune étude qui analyse la relation entre homophobie et citoyenneté n'a été trouvée. Pourtant, les demandes du mouvement et le rapport du GGB démontrent que les personnes LGBT au Brésil doivent composer avec une violence telle qu'un membre de leur communauté est tué toutes les 19 heures.

\section{La violence qui maintient les systèmes de pouvoir}

Selon la sociologue britannique Walby (2009), bien que l'analyse de la violence et son influence par rapport au bien-être des personnes, aux régimes d'inégalité et dans d'autres domaines institutionnels soit importante, elle est sous-estimée dans la théorie sociale, qui la relègue la plupart du temps à la criminologie. L'auteure voit la violence comme un phénomène social qui constitue un système social, un domaine institutionnel fonctionnant en parallèle à l'économie, à la politique et à la société civile. Elle attire l'attention sur les formes de violence qui ne sont pas criminalisées, mais qui sont généralisées, et conséquemment admises par l'État, telles que la violence exercée contre les femmes et les groupes ethniques. Les types de violence qui sont acceptés par l'État varient selon le contexte géopolitique. $\mathrm{Au}$ Brésil, les attaques contre des groupes ethniques sont presque inexistantes, tandis que l'homophobie présente des taux élevés et n'est pas criminalisée.
Walby établit une relation entre la consistance du régime démocratique et le niveau de violence. Ainsi, les niveaux de violence plus élevés existeraient dans des pays avec un haut niveau d'inégalité sociale et une démocratie fragile. Le Brésil détient les deux caractéristiques mentionnées par la sociologue. Le régime démocratique a été rétabli depuis à peine trente-trois ans, les niveaux d'inégalité sociale qui étaient en baisse pendant les premières années de gouvernement du Parti des Travailleurs s'aggravent depuis les dernières années et les indices de violences sont accablants. Des données ${ }^{7}$ démontrent notamment qu'en 2016, 62517 homicides ont été commis au pays.

La sociologue rejette la théorie attribuant aux personnes défavorisées sur le plan socio-économique l'exécution de la plupart des crimes. Sa lecture de la violence est intersectionnelle. Les inégalités doivent être pensées de façon plus complexe, au-delà de la différence de classe. Ainsi, quand d'autres inégalités sont mises en évidence, les formes interposées de la violence interpersonnelle, surtout celles non criminalisées, deviennent plus significatives. Pour Walby, la violence est souvent utilisée par les groupes dominants comme une forme de pouvoir instrumental visant à maintenir les hiérarchies de genre, d'ethnie, raciales, nationales et sexuelles. L'implication de la violence dépend de sa réglementation et de la résilience des victimes potentielles.

\section{La violence faite aux personnes noires}

Les chiffres démontrent que la violence au Brésil est un problème qui affecte l'ensemble de la société. Cependant, il est important de

7 Information du « Atlas da Violência », site web coordonné par l'organisme gouvernemental brésilien IPEA - Institut de recherche économique et appliquée. https:// bit.ly/2yBW1V1 
souligner que certains types de violence sont dirigés vers un groupe spécifique de la population et sont socialement tolérés, justement pour le maintien de l'hégémonie, comme l'affirme Walby. C'est le cas du féminicide, de la violence raciale et de l'homophobie. Dans certaines circonstances, même l'État peut déployer une force excessive. Nous parlons notamment de la violence policière exercée à l'endroit des personnes noires aux États-Unis et au Brésil. Dans son article de 2015, l'anthropologue américaine Christen A. Smith se base sur les données de la violence policière faite aux personnes noires dans ces deux pays pour soutenir que la race, en tant que construction sociale, historique et politique, définit, encore de nos jours, l'expression de la citoyenneté, le type de citoyenneté qui est mise en pratique ainsi que la reconnaissance elle-même des sujets qui sont considérés en tant que citoyens.

Smith affirme qu'au Brésil et aux ÉtatsUnis, les personnes noires ne sont des citoyens qu'au sensu stricto (2015, p. 385). Pour constituer son argument, l'auteure a réalisé une analyse qualitative de l'expérience nationale. Le postulat que tous les citoyens bénéficient d'une protection égale en vertu de la loi ne s'applique pratiquement pas aux personnes noires de ces pays. L'inclusion existe en théorie, mais n'est pas mise en pratique par l'État. Pour l'auteure, ce qui prouve son argument est la manière indiscriminée dont les personnes noires sont traitées par les forces de l'ordre de l'État. En effet, les Noirs américains et brésiliens sont tués, battus, torturés et violés par ce dernier, et ce, en toute impunité. Parfois, même ceux qui sollicitent l'assistance de la police sont assassinés pour en avoir demandé. Bien que récent, son article fait écho à une recherche antérieure qui a démontré la même réalité au Brésil, et cela depuis au moins 1988 (MITCHELL et WOOD, 1999).

\section{Le féminicide}

La violence faite aux femmes est une autre catégorie de violence qui est dirigée spécifiquement vers une partie de la population. Malheureusement, elle s'avère si répandue qu'elle a même un nom pour la définir : il s'agit du féminicide. La mission des États-nations consiste à exprimer la volonté de leurs citoyens et à défendre les intérêts de la nation (BRUBAKER, 1997). C'est dans ce présupposé que se tient l'exclusion de non-citoyens au suffrage universel. Or, dans plusieurs pays sur la planète, les femmes se sont battues pour avoir un tel droit. Si les premières étapes pour l'obtention d'une citoyenneté à part entière ont débuté par la lutte pour le droit de vote, actuellement, leurs droits continuent d'être lésés par la persistance de la violence perpétrée à leur égard (FRANZWAY, 2016).

L'analyse de Soriano (2014) fait écho à celle de Walby, car elle voit la violence faite aux femmes non seulement comme le résultat des inégalités et des discriminations auxquelles elles sont confrontées, mais avant tout comme un instrument pour maintenir, produire et reproduire ces inégalités et discriminations. Franzway (2016) s'aligne sur les autres auteures mentionnées. Elle démontre que la citoyenneté a toujours été définie et exercée en relation au genre et que cela fait partie de la politique sexuelle de la citoyenneté. Pour cette raison, l'auteure défend que la violence à l'égard des femmes pourrait diminuer si les femmes étaient des citoyennes placées sur un pied d'égalité avec les hommes, si elles avaient accès au pouvoir de l'État et aux droits politiques, sociaux et civils pour garantir des réponses efficaces et équitables aux violences qu'elles subissent. Dans ce même article, Franzway mobilise l'auteure Susan James (1992). Cette dernière défend que pour pouvoir participer à la démocratie, les citoyens doivent 
être protégés contre les contraintes coercitives, les violences corporelles ou bien la menace à celle-ci.

Si la violence perpétrée contre les personnes noires vient surtout de l'État (SMITH, 2015; MITCHELL et WOOD, 1999), celle faite aux femmes provient plus souvent d'un homme de leur entourage et bénéficie généralement de l'impunité (SORIANO, 2014). Malgré certaines variations sur la façon dont la violence est exercée, les analyses sur la violence raciale, sur le féminicide et sur leur relation avec l'exercice de la pleine citoyenneté qui ont été mentionnées démontrent que la différence de traitement que certaines personnes reçoivent de l'État est incompatible avec l'idée de citoyenneté universelle. L'homophobie est un type de violence spécifique qui correspond aux autres types de violence analysés. Il s'agit d'une violence interpersonnelle non criminalisée, destinée aux personnes de la diversité sexuelle et normalement commise en toute impunité.

\section{L'homophobie : une barrière au vécu citoyen}

Nous pouvons dire que la motivation du féminicide vient en partie de ce qui stimule l'homophobie. Le féminicide est le résultat des modèles de pensée fondés sur l'idée que les femmes sont inférieures aux hommes, ce qui génère des attitudes machistes, dévalorisantes et la haine envers les femmes (PERES et al., 2018). Les racines de l'homophobie sont liées à la misogynie et au sexisme. Pour les hommes gays, c'est le manque d'une posture perçue comme étant masculine qui motive l'intimidation à caractère homophobe, c'est le rejet des traits considérés comme féminins chez les hommes (BORILLO, 2001; WELZER-LANG et al., 1995). Pour les femmes lesbiennes, c'est tout à fait le contraire. Dans ce cas, ce qui motive la haine est le rejet de ce qui est consi- déré comme étant masculin chez les femmes. Les deux formes de violence se basent alors sur l'infériorisation des personnes selon leur identité de genre, leur expression de genre et pour l'homophobie, on ajoute l'orientation sexuelle.

L'ensemble des rapports sociaux et structures qui génèrent et supportent la croyance selon laquelle les personnes LGBTQ ont une orientation sexuelle et une expression de genre qui ne cadrent pas dans les normes existantes est connu comme l'hétéronormativité (BASTIEN CHARLEBOIS, 2011; CHAMBERLAND et PAQUIN, 2005). Il est socialement attendu que les personnes soient hétérosexuelles et que leur expression de genre se conforme à leur sexe assigné à la naissance. Autrement dit, une personne qui a été assignée comme homme à la naissance doit agir et posséder des caractéristiques masculines, et celles assignées comme femme à la naissance doivent avoir une allure féminine. Cette norme hétérosexuelle et cisgenrée qui promeut l'hétérosexualité comme la seule orientation sexuelle socialement acceptable classe les autres orientations et les expressions de genre diverses comme secondaires. Or, l'idée qui vient d'emblée avec cette norme est que ceux qui ne la suivent pas sont secondaires, marginaux et inférieurs. Il appert que la violence homophobe entre dans la définition de Walby (2005), comme étant une forme de pouvoir instrumental visant à maintenir la hiérarchie des sexualités.

Un autre constat en lien avec Walby (2005) est le caractère socialement acceptable des violences non criminalisées. Des 445 crimes commis contre la population LGBT au Brésil en 2017, moins de $10 \%$ des cas ont eu une enquête policière menée (MOTT et al., 2018). À la lumière de ces statistiques, le postulat que tous les citoyens bénéficient d'une protection égale en vertu de la loi devient discutable et semble ne pas s'appliquer aux personnes de la diver- 
sité sexuelle. Ce postulat a d'ailleurs été utilisé par Smith (2015) pour contester l'existence d'une réelle citoyenneté des personnes noires au Brésil et aux États-Unis.

Dans un article de 2010, Glenn défend que la citoyenneté, qui signifie l'appartenance à sa communauté, se réalise par la reconnaissance du membership de la part d'autres membres qui la constituent. L'auteure soutient que ce sont les membres de la communauté qui définissent les bénéficiaires des droits civils, politiques et sociaux, et cela se fait à travers l'accord ou le refus de la reconnaissance de ces bénéfices. Malgré les taux élevés de crime violent contre la population LGBT et les demandes de cette population pour la criminalisation de ce type de crime, les parlementaires brésiliens refusent d'approuver les projets de loi qui ont été élaborés à cet effet. À titre d'exemple, un projet de $2006^{8}$ a été archivé et un autre projet de $2016^{9}$ n'a pas encore été voté. Cela démontre que la société brésilienne ne voit pas les membres de la communauté LGBT sur un pied d'égalité, ils ne sont pas reconnus comme méritant les mêmes bénéfices que possèdent les citoyens hétérosexuels. Ils ne sont que citoyens partiaux, car ils ne bénéficient pas de la citoyenneté civile, celle que Marshall a conceptualisée comme le droit à la justice (RICHARDSON, 1998).

Ainsi, un parallèle peut être établi entre le rôle de la violence homophobe, du féminicide et du racisme dans la construction de la citoyenneté. Nous ne faisons pas l'hypothèse selon laquelle les manifestations et les conséquences de ces trois types de violence sont les mêmes. Cependant, en ce qui a trait à la construction de la citoyenneté, ces violences produisent le même effet, soit la construction d'une barrière qui empêche l'accès au membership que la citoyenneté devrait garantir. Si la partici-

$8 \quad \mathrm{PL} 122$

9 SUG 5/2016 pation à la démocratie émane seulement des citoyens libres des contraintes coercitives, de violences corporelles ou bien de la menace à celle-ci (JAMES, 1992), les personnes soumises à un danger imminent, comme cela est le cas des LGBT, ne peuvent pas y participer. En nous alignant au processus de construction des hiérarchies raciales identifié par Smith (2015), nous appréhendons la construction de l'hétérosexualité comme la seule orientation acceptable et sa conséquence flagrante - l'homophobie - comme l'expression de la citoyenneté et la reconnaissance des sujets qui sont considérés en tant que citoyens.

\section{Conclusion}

Ce travail a retracé les origines de l'étude de la citoyenneté depuis l'œuvre de Marshall et son rôle en tant que fondement sociologique dans la réflexion et l'analyse des multiples façons d'explorer la citoyenneté, parmi lesquelles on trouve la citoyenneté sexuelle. Après l'exposition du concept de citoyenneté sexuelle, nous avons démontré que celui-ci a été construit en ayant comme assise le contexte du Nord global. Deux hypothèses principales ont alors guidé l'élaboration de cet article. La première hypothèse postule que la construction de la citoyenneté sexuelle ne se fait pas de la même manière au Nord et au Sud globaux. La deuxième repose sur le fait que la violence homophobe dans le contexte brésilien est, par sa récurrence, un élément primordial dans l'analyse de cette catégorie dans ce pays. Ainsi, nous avons réalisé une brève exposition comparative des demandes des mouvements sociaux des gays et des lesbiennes aux États-Unis et au Brésil. La finalité de cette comparaison a été la mise en évidence que chaque contexte social et politique génère des demandes distinctes et que, par conséquent, la construction de la citoyenneté sexuelle ne peut se faire de la même manière. 
Pour démontrer que l'homophobie empêche l'accès aux bénéfices qui font normalement partie du statut de citoyen, nous avons eu recours à des œuvres qui traitent de la relation entre la violence et la démocratie, ainsi qu'à des articles qui définissent le féminicide et le racisme comme barrières d'accès à la pleine citoyenneté. Finalement, nous avons établi un parallèle entre ces études et la violence homophobe, dans le but de prouver l'hypothèse de départ qui voit les enjeux de l'homophobie au Brésil comme une entrave au membership que la citoyenneté est censée garantir. Le manque d'études sur le cas brésilien qui mettent en exergue la violence homophobe et la citoyenneté sexuelle est problématique, vu la dimension de la violence et la négligence délibérée de la part des politiciens et du restant de la société civile. Si ce champ d'études semble saturé au Nord global, cela ne s'avère pas le cas pour les divers contextes des pays du Sud.

\section{Références}

AMMATURO, F. R. The 'Pink Agenda': questioning and challenging european homonationalist sexual citizenship. Sociology, v. 49, n. 6, p. 1151-1166, 2015.

ARMSTRONG, E. Forging gay identities: Organizing sexuality in San Francisco, 1950-1994. Chicago: University of Chicago Press, 2002.

BASTIEN CHARLEBOIS, J. Au-delà de la phobie de l'homo: quand le concept d'homophobie porte ombrage à la lutte contre l'hétérosexisme et l'hétéronormativité. Reflets: Revue d'intervention sociale et communautaire, v. 17, n. 1, p. 112-149, 2011.

BORRILLO, D. L'homophobie: «Que sais-je?». France: Presses Universitaires de France, 2001.

BRUBAKER, R. Citoyenneté et nationalité en France et en Allemagne. Paris: Paris Belin, 1997.

CANABARRO, R. História e direitos sexuais no Brasil: o movimento LGBT e a discussão sobre a cidadania. In: CONGRESSO INTERNACIONAL DE HISTÓRIA REGIONAL, 2., 2013. Passo Fundo, RS. Anais eletrônicos...
Passo Fundo, RS: Universidade de Passo Fundo, 2013.

CARRARA, S. Anthropology and the Process of the Construction of Homosexual Citizenship in Brazil. Cadernos Pagu, n. 47, p. 445-482, 2016.

CARTER, David. Stonewall: The riots that sparked the gay revolution. New York: St. Martin's Press, 2004.

CHAMBERLAND, L.; PAQUIN, J. Penser le genre, penser l'hétérosexualité: un défi pour la troisième vague du féminisme. Dialogues sur la troisième vague féministe, Montréal, Les éditions du remue-ménage, p. 119-131, 2005.

D'EMILIO, John. Sexual politics, sexual communities. Chicago: University of Chicago Press, 1998.

DEMCZUK, Irène; REMIGGI, Frank W. (Orgs.). Sortir de l'ombre. Histoires des communautés lesbienne et gaie de Montréal. Montréal: VLB Éditeur, 1998.

DUGGAN, Lisa. The twilight of equality?: neoliberalism, cultural politics, and the attack on democracy. Boston: Beacon Press, 2004.

EL-TAYEB, F. European others: queering ethnicity in post-racial Europe. Minneapolis: University of Minnesota Press, 2011.

EVANS, D. T. Sexual citizenship: the material construction of sexualities. London: Routledge, 1993.

FACCHINI, R.; SIMÕES, J. A. Na trilha do arco-íris: do movimento homossexual ao LGBT. São Paulo: Perseu Abramo, 2009.

FERNANDES, E. Ativismo homossexual indígena: uma análise comparativa entre Brasil e América do Norte. Dados - Revista de Ciências Sociais, v. 58, n. 1, p. 257-294, 2015.

FILHO, R. E. Corpos brutalizados: conflitos e materializações nas mortes de LGBT. Cadernos Pagu, v. 0, n. 46, p. 311-340, 2016.

FRANZWAY, S. The sexual politics of citizenship and violence. Women's Studies International Forum, v. 58, p. 18-24, 2016.

GLENN, E. N. Citizenship and Inequality: Historical 
and Global Perspectives. Social Problems, v. 47, n. 1, p. 1-20, 2000.

GLENN, E. N. Constructing citizenship: exclusion, subordination and resistance. American Sociological Review, v. 76, n. 1, p. 1-24, 2011.

GROSSI, M. P. Aula 1: Teoria queer e questões trans. In: Curso de curta duração em gênero e feminismo. Florianópolis: UFSC, 2015.

JAMES, S. The good-enough citizen: citizenship and independence. In: BOOKS, G.; JAMES, S. (Eds.). Beyond equality and difference: citizenship, feminist politis and female subjectivity. London: Routledge,1992. p. 47-67

HALL, S.; HELD, D. Left and Rights. Marxism Today, p. 16-23, 1989.

HIGGINS, R. La régulation sociale de l'homosexualité. De la répression policière à la normalisation. In: CORRIVEAU, Patrice; DAOUST, Valérie. (Eds.). La régulation sociale des minorités sexuelles. L'inquiétude de la différence. Québec: Presses de l'Université du Québec, 2011. p. 67-101.

IRINEU, A. B. Homonacionalismo e cidadania LGBT em tempos de neoliberalismo: dilemas e impasses às lutas por direitos sexuais no Brasil. Revista em Pauta, v. 12, n. 34, p. 155-178, 2014.

ISIN, E. F.; TURNER, B. S. (Eds.). Handbook of citizenship studies. London: Sage, 2002.

ISIN, E.; WOOD, P. Citizenship and identity. London: Sage, 1999.

KAHLINA, K. Local histories, European LGBT designs: Sexual citizenship, nationalism, and "Europeanisation" in post-Yugoslav Croatia and Serbia. Women's Studies International Forum, v. 49, p. 73-83, 2015.

LISTER, R. Sexual Citizenship. In: ISIN, E. F.; TURNER, B. S. (Eds.). Handbook of citizenship studies. London: Sage, 2002. p. 191-208.

MARSHALL, T. H. Citizenship and social class. Cambridge: Univeristy Press, 1950.

MCGEE, R. Power, Violence, Citizenship and Agency. IDS Bulletin, v. 45, n. 5, p. 36-47, 2014.
MIÑOSO, Y. E. El futuro ya fue: una critica a la idea del progreso en las narrativas de liberación sexo-genérica y queer identitarias en Abya Yala. In: FERRERA-BALANQUET, R. M. (Comp.). Andar erótico decolonial. Buenos Aires: Ediciones del Signo, 2015. p. 21-35.

MITCHELL, M. J.; WOOD, C. H. Ironies of citizenship: skin color, police brutality, and the challenge to democracy in Brazil. Social Forces, v. 77, n. 3, p. 10011020, 1999.

MOTT, L. A construção da cidadania homossexual no Brasil. Democracia Viva, v. 25, n. 99, p. 98-102, 2005.

MOTT, L.; MICHELS, E.; PAULINHO. Relatório 2017 Assassinatos de LGBT no Brasil. Bahia: Grupo Gay da Bahia - GGB, 2018.

NOGUEIRA, C.; OLIVEIRA, J. M. de. Estudo sobre a discriminação em função da orientação sexual e da identidade de género. Lisboa: Comissão para a Cidadania e Igualdade de Género, 2010.

OLIVEIRA, J. M. de. Cidadania sexual sob suspeita: uma meditação sobre as fundações homonormativas e neo-liberais de uma cidadania de "consolação" [Sexual citizenship under suspicion: a meditation on the homonormative and neoliberal foundations of a "consolation" citizenship]. Psicologia \& Sociedade, v. 25, n. 1, p. 68-78, 2013.

PATERNOTTE, D.; TREMBLAY, M. The Ashgate research companion to lesbian and gay activism [ressource électronique]. Abingdon, Oxon: Routledge, 2016.

PELÚCIO, L. Subalterno quem, cara pálida? Apontamentos às margens sobre pós-colonialismos, feminismos e estudos queer. Revista Semestral do Departamento e do Programa de Pós-Graduação em Sociologia da UFSCar, v. 2, n. 2, p. 395-418, 2012.

PEREIRA, P. P. G. Queer decolonial: quando as teorias viajam. Revista Semestral do Departamento e do Programa de Pós-Graduação em Sociologia da UFSCar, v. 5, n. 2, p. 411-437, 2015.

Peres, M. C. C.; SOARES, S. F.; DIAS, M. C. Dossiê sobre lesbocídio no Brasil de 2014 a 2017. Rio de Janeiro: Livros Ilimitados Editora e Assessoria, 2018. 
PLUMMER, K. Telling sexual stories: power, change and social worlds. London: Routledge, 1995.

PLUMMER, K. Intimate citizenship in an unjust world. In: ROMERO, M.; MARGOLIS, E. (Eds.). The blackwell companion to social inequalities. Malden: Blackwell Publishing Ltd., 2005. p. 77-94.

RAGIN, C. C. The comparative method: moving beyond qualitative and quantitative strategies. Oakland, California: University of California Press, 2014.

REA, C. A.; AMANCIO, I. M. S. Sexualidades dissidentes e teoria queer pós-colonial: o caso africano. Revista Epistemologias do Sul, v. 1, n. 1, p. 145-165, mai. 2017.

REA, C. A.; AMANCIO, I. M. S. Descolonizar a sexualidade: teoria queer of colour e trânsitos para o Sul. Cadernos Pagu, n. 53, p. 1-38, 2018.

RICHARDSON, D. Sexuality and citizenship. Sociology, v. 32, n. 1, p. 83-100, 1998.

RICHARDSON, D. Constructing sexual citizenship: theorizing sexual rights. Critical Social Policy, v. 20, n. 1, p. 105-135, 2000.

RICHARDSON, D. Rethinking sexual citizenship. Sociology, v. 51, n. 2, p. 208-224, 2017.

RITTER, P. D. Comparative historical analysis. In: DELLA PORTA, D. (Ed.). Methodological practices in social movement research. United Kingdom: Oxford University Press, 2014. p. 97-116.

ROCHE, M. Social Citizenship: grounds of social change. In: ISIN, E. F.; TURNER, B. S. (Eds.). Handbook of citizenship studies. London: Sage, 2002. p. 69-87.

ROSENEIL, S.; STOILOVA, M. Heteronormativity, intimate citizenship and the regulation of same-sex sexualities in Bulgaria. In: KULPA, R.; MIZIELIÂNSKA, J. (Eds.) De-centring western sexualities: Central and Eastern European perspectives. Furnham: Ashgate, 2011. p. 167-190.

SILVÉRIO TREVISAN, J. Devassos no paraíso. 3. ed. Rio de Janeiro; Sao Paulo: Record, 2000.

SMITH, C. A. Blackness, citizenship, and the transnational vertigo of violence in the Americas. American Anthropologist, v. 117, n. 2, p. 384-387, 2015.

SORIANO, M. Citoyenneté et formes de violence. La violence de genre en Amérique latine. Caravelle, p. 9-20, 2014.

SOUSA, B. A. De la clandestinité à la fierté: l'approppriation de l'espace urbain par la communauté gay. 2016. 125 f. (Mestrado em Sociologia) - Maîtrise en sociologie avec mémoire, Université Laval, Québec, 2016.

SPIVAK, G. C. Les subalternes peuvent-elles parler? Paris: Éditions Amsterdam, 2009.

TREVISAN, J. S. Devassos no paraíso: a homossexualidade no Brasil, da colônia à atualidade. 4. ed. Rio de Janeiro: Objetiva, 2018.

WALBY, S. Globalization and inequalities complexities and contested modernities. Los Angeles: Sage 2009.

WEEKS, Jeffrey. The sexual citizen. Theory, Culture \& Society, Londres, v. 15, n. 3-4, p. 35-52, ago. 1998.

WELZER-LANG, D.; DUTEY, P.-J.; DORAIS, M. La peur de l'autre en soi: du sexisme à l'homophobie. Montréal: Vlb éditeur, 1995.

Recebido em: 25/02/2019

Aprovado em: 22/05/2019

Barbara Andrade de Sousa Doutouranda em sociologia na Université du Québec à Montréal. Mestre em sociologia pela Université Laval, Québec. Graduação em Comunicação Social - UniCeub, Brasília. Auxiliar de pesquisa no projeto SAVIE-LGBTQ https://savie-Igbtq.uqam.ca/ e-mail: sousa.barbara_andrade@courrier.uqam.ca

4819, Montée Saint-Hubert, Saint-Hubert, QC, J3Y 1V4. Telefone: 1 (418) 261-7411 